\title{
Prevalence of and Factors Associated with School Bullying in Students with Autism Spectrum Disorder: A Cross-Cultural Meta-Analysis
}

\author{
Inhwan Park ${ }^{1}$, Jared Gong ${ }^{2}$, Gregory L. Lyons ${ }^{3}$, Tomoya Hirota ${ }^{4}$, Michio Takahashi ${ }^{5,6}$, Bora Kim , \\ Seung-yeon Lee ${ }^{1}$, Young Shin Kim ${ }^{3,4}$, Jeongsoo Lee ${ }^{1}$, and Bennett L. Leventhal ${ }^{3,4}$ \\ ${ }^{1}$ Department of Psychology, Ewha Womans University, Seoul, Korea; \\ ${ }^{2}$ Department of Psychiatry and Behavioral Sciences, Stanford University, Stanford, CA, USA; \\ ${ }^{3}$ Center for ASD and Neurodevelopmental Disorders, University of California, San Francisco, CA, USA; \\ ${ }^{4}$ Department of Psychiatry and Behavioral Sciences, University of California, San Francisco, CA, USA; \\ ${ }^{5}$ Research Center for Child Mental Development, Hirosaki University, Hirosaki, Aomori, Japan; \\ ${ }^{6}$ Department of Clinical Psychological Science, Hirosaki University, Hirosaki, Aomori, Japan.
}

Through this meta-analysis, we sought to examine the prevalence of, risks for, and factors associated with bullying involvement (victimization, perpetration, perpetration-victimization) among students with autism spectrum disorder (ASD). Additionally, we attempted to examine sources of variance in the prevalence and effect sizes of bullying in students with ASD across studies. Systematic database and literature review identified 34 relevant studies (31 for Western countries, three for Eastern countries). Pooled prevalence estimates for victimization, perpetration, and perpetration-victimization in general were $67 \%$, $29 \%$, and $14 \%$, respectively. The risk of victimization in students with ASD was significantly higher than that in typically developing students and students with other disabilities. Further, deficits in social interaction and communication, externalizing symptoms, internalizing symptoms, and integrated inclusive school settings were related to higher victimization, and externalizing symptoms were related to higher perpetration. Finally, moderation analyses revealed significant variations in the pooled prevalences thereof depending on culture, age, school settings, and methodological quality and in the pooled effect sizes according to publication year and methodological quality. Our results highlight needs for bullying intervention for students with ASD, especially those who are younger, are in an inclusive school setting, and have higher social difficulties and externalizing/internalizing symptoms; for intensive research of bullying experiences among students with ASD in Eastern countries; and for efforts to improve the methodological quality of such research.

Key Words: Students with ASD, bullying, prevalence, associated factors, cultural difference, methodological quality

Playing a key role in the development of children and adolescents, peer relationships provide the foundation for the development of social and verbal skills, cooperative learning, and social supports. ${ }^{1}$ Affecting an estimated $45 \%$ of students, ${ }^{2}$ bullying, a form of peer victimization/abuse, is the most common form of violence among children and adolescents and interferes with achieving critical developmental tasks.

Bullying affects both typically developing students and those with developmental disabilities. A review of 32 studies on students with disabilities reported that the prevalence of victim-

Received: May 29, 2020 Revised: September 6, 2020 Accepted: September 17, 2020

Corresponding author: Young Shin Kim, MD, PhD, Department of Psychiatry and Behavioral Sciences, Center for ASD and Neurodevelopmental Disorders, University of California, Langley Porter Psychiatric Institute, LP-377, 401 Parnassus Ave, Box 0984, San Francisco, CA 94143-0984, USA.

Tel: 1-415-502-2999, Fax: 1-415-476-7320, E-mail: YoungShin.Kim@ucsf.edu

-The authors have no potential conflicts of interest to disclose.

(C) Copyright: Yonsei University College of Medicine 2020

This is an Open Access article distributed under the terms of the Creative Commons Attribution Non-Commercial License (https://creativecommons.org/licenses/by-nc/4.0) which permits unrestricted non-commercial use, distribution, and reproduction in any medium, provided the original work is properly cited. 
ization and perpetration ranges from $9 \%$ to $100 \%$ and from $6 \%$ to $83 \%$, respectively. ${ }^{3}$ Furthermore, a number of studies have indicated that students with disabilities are at greater risk for experiencing bullying than typically developing students. ${ }^{4,5}$ Students with disabilities may be selected as easy targets because bullies see them as unable to protect themselves or their behavioral problems may make them vulnerable for peer victimization. On the other hand, individuals with disabilities may be seen as bullies, due to emotional regulation difficulties and/or inappropriate coping strategies. ${ }^{3,4}$ With research suggesting that the characteristics of disabilities act as risk factors for bullying, ${ }^{6,7}$ it is not surprising that students with disabilities are at increased risk for bullying involvement.

Among neurodevelopmental disorders, students with autism spectrum disorder (ASD) appear to be particularly vulnerable to bullying involvement due to their deficits in social communication, as well as difficulty with empathy. ${ }^{8-10}$ Similarly, behavioral difficulties could be caused by hyper-responsiveness to sensory stimulus or insistence on sameness, which are known risk factors for bullying. ${ }^{11-13}$ A recent meta-analysis of bullying prevalence studies in students with ASD reported the prevalences of bullying involvement as victims, bullies, and bully-victims as $44 \%, 10 \%$, and $16 \%$, respectively. ${ }^{14}$ The prevalences of victimization and perpetration in students with ASD were significantly higher than those in typically developing students, as well as in those with other disabilities. ${ }^{15,16}$ Further studies have found that gender, ${ }^{17,18}$ age, ${ }^{17,19}$ severity of ASD symptoms, ${ }^{19,20}$ externalizing and internalizing symptoms, ${ }^{21,22}$ and school settings $^{19,23}$ all have significant associations with bullying in the ASD population.

Only one meta-analysis study of bullying research in students with ASD has been conducted. ${ }^{14}$ It provided valuable information on pooled bullying prevalences (bullying as a whole, as well as specific types of bullying, including verbal, physical, and relational), risks of bullying in students with ASD in comparison to typically developing students, and sources of variation in bullying prevalence across different studies (geographic location, school settings, information source, type of measures, assessment time-frame, and bullying frequency criterion). However, there are informational gaps in several areas in the meta-analysis study. First, no data on cyber-bullying experiences for children with ASD were integrated, despite data suggesting that students with ASD are likely to suffer cyber-bullying. ${ }^{24}$ Also, students with other disabilities tend to experience bullying more frequently than typically developing students. ${ }^{4,5}$ However, there is a lack of information on whether students with ASD experience bullying differently from those with other disabilities. Additionally, there is considerable variability in bullying prevalences, and a variety of factors may account for the variance. In addition to geographic location, school settings, information source, type of measures, assessment time-frame, and bullying frequency criterion, ${ }^{14}$ differences in research methods can cause variations in study results (e.g., reliability and validity of bullying measurement, sampling bias, etc.). Also, it has been suggested that culture affects study results because individual thoughts and behaviors are influenced by culture. In Western culture, there is a general expectation that individuals will be self-reliant and independent from parents (individualistic). In contrast, in Eastern culture, there is an emphasis on cohesiveness among individuals (collectivistic), ${ }^{25}$ respects for elders, and compliance of students toward adults. ${ }^{26}$ Those cultural characteristics may affect a bystander's behaviors and coping strategies, such as seeking help from adults, which can lead to different patterns of bullying in Western and Eastern cultures.

Meta-analysis is a statistical approach for integrating results from multiple studies in an effort to increase study power and overcome the statistical weaknesses of small sample sizes in individual studies. It also improves estimates of effect sizes and helps resolve uncertainty when results disagree. ${ }^{27}$ We conducted a meta-analysis with the following objectives: the first objective was to examine pooled prevalence estimates of bullying involvement in relation to victimization, perpetration, and perpetration-victimization in general, physical, verbal, relational, and cyber forms. The second objective was to investigate whether pooled prevalence estimates of bullying among students with ASD differ from other disabilities and typically developing students. The third objective was to identify factors associated with bullying in students with ASD across multiple studies. The final objective was to examine whether variations in pooled prevalence estimates and pooled effect sizes for students with ASD are affected by study-related characteristics, including publication year, culture (Western culture, Eastern culture), sample characteristics (gender, age, school settings), bullying measurement (measurement method, bullying frequency criteria, assessment time-frame, respondents), and methodology quality.

\section{INCLUSION CRITERIA}

Studies that meet all the eligibility criteria were included in this meta-analysis: 1) studies must include students diagnosed with ASD [autism, Asperger syndrome, pervasive developmental disorder-not otherwise specified (PDD-NOS)]; 2) studies must have information on prevalence estimates of school bullying, factors correlated with bullying, predictors of bullying, and/or outcomes of bullying; 3) study is designed as a cohort, crosssectional, or case-control study, excluding qualitative, longitudinal, intervention, and theory studies; 4) studies are published in English, Japanese, or Korean; and 5) studies were published in peer reviewed journals by January 2018.

\section{SEARCH AND SELECTION OF STUDIES}

Electronic databases of social science and psychiatry literature were utilized for literature searches in 1) English (Academic 
Search Complete, CINAHL Plus with Full Text, Education Sources, E-Journals, ERIC, Medline, PsycARTICLES, PsychINFO, and SocINDEX with Full Text); 2) Japanese [Medical Online, CiNii, J-Stage, and Japan Medical Abstracts Society (Ichusi)]; and 3) Korean (DBpia, KISS, RISS, KYOBO Scholar, eArticle, and Newnonmun). Keywords for the literature search included all possible combinations of (a) autis* OR asperger OR pervas* developm* dis* OR PDD-NOS, (b) bull* OR victim* OR perpetrat* OR teas* or violen*, and (c) school* OR education* OR class* in the English databases; (a) autism OR ASD OR Asperger OR neurodevelopmental disorder OR pervasive developmental disorder, (b) ijime, and (c) school OR class in the Japanese databases; and (a) autism, Asperger, PDD-NOS and (b) peer-bullying, bullying, Wang-tta, school violence in the Korean databases. The database searches yielded 848 articles in English, 312 articles in Japanese, and 18 articles in Korean. Additionally, review of references in previous review/meta-analysis studies $^{14,28-32}$ yielded 78 articles in English and seven articles in Korean.

We reviewed the titles and abstracts of each study to determine eligibility for inclusion in this meta-analysis. This resulted in 62 articles in English, seven articles in Japanese, and nine articles in Korean. After a full-text review, 34 articles in English, one article in Japanese, and no articles in Korean remained (Fig. 1).

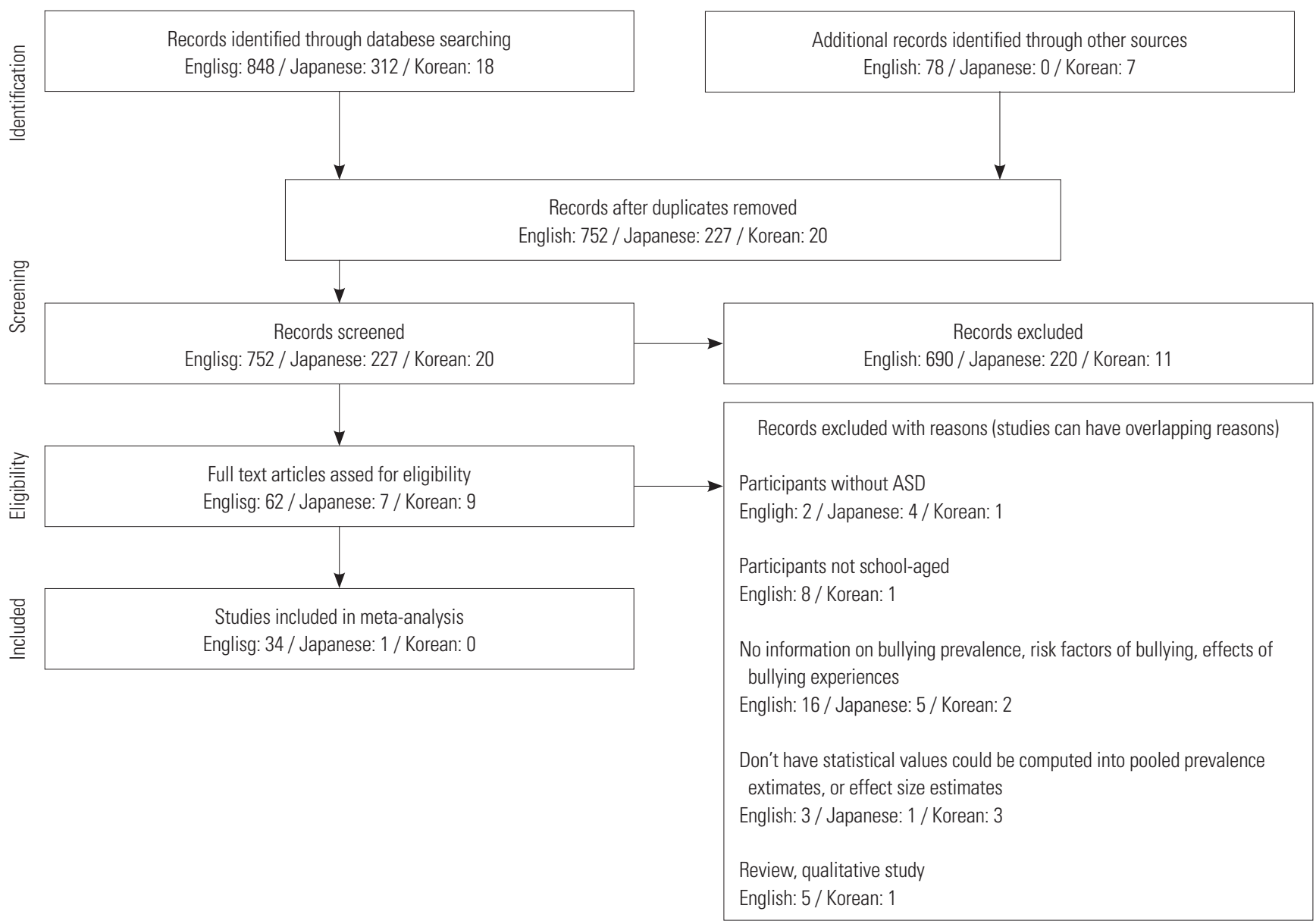
cedures for meta-analysis. The database searches and the literature review were conducted independently. In all steps, consensus decisions were made by two authors per each language, and discrepancies were resolved by discussion. Percentage agreement between the authors was $93 \%$ for English articles and $100 \%$ for Japanese and Korean studies. Supplementary Table 1 (only online) ${ }^{33-56}$ for a final list of the reviewed studies.

\section{ASSESSMENT OF METHODOLOGICAL QUALITY OF STUDIES}

The methodological quality of each study was assessed with the Appraisal Tool for Cross-Sectional Studies (AXIS). ${ }^{57}$ AXIS covers three domains: reporting quality ( 7 items), study design quality (7 items), and biases (selection, information, non-response; 6 items). Of these, the 13 items for study design quality and biases were used to assess the methodological quality of each study, and the seven items from reporting quality (how well methods, results, and discussion are described well) were excluded because they were irrelevant to methodological quality. AXIS only addresses selection, information, and non-response biases, but
The first author provided other authors with a manual of pro-

Fig. 1. Flow diagram illustrating search and selection of studies. 
not confounding bias, a major threat to the study validity. Item 11 of the Critical Appraisal Checklist for Cross-Sectional Study ${ }^{58}$ was added to measure confounding bias. This item asks the question: "Could there be confounding factors that haven't been accounted for?".

Selection bias occurs when every unit in the sample frame does not have the same probability of being selected. Information bias occurs when systematic differences in the accuracy of data lead to a distortion in research results. Non-response bias occurs when the non-responders are systematically different from responders in the sample. Confounding bias occurs when an extraneous variable distorts the association between an independent variable and a dependent variable.

In the end, 14 items were used to assess the methodological quality of the included studies. All items were coded as 1 when a study satisfied a qualitative standard or as 0 when a study did not satisfy a qualitative standard or when assessment was not possible (unknown). Scores ranged from 0 to 14, with higher scores indicating better methodological quality. Two authors assessed cooperatively the methodological quality for each study and reached consensus decisions.

\section{DATA CODING}

Information from each study was collected using an electronic data extraction sheet developed for this review. In each study, the following items were coded: publication year, culture (Western culture/Eastern culture), gender (percentage of boys in the sample), age (childhood: 5-12 years; adolescents: 13-22 years; mixed:5-22 years), school settings (inclusive/ segregated/ mixed), measurement method (questionnaire/interview/mixed), bullying frequency criterion (dichotomous score: experience bullying yes or no/cut-off score: experience more than once a week, or twice a week, or once a month, or twice a month, or four times a year), assessment time-frame (month: last month or months/ year: last or current year/life-time), and respondents (self/parent/teacher/peer). Age was coded based on both the age mean and the age range of the sample. Culture was classified as Western or Eastern culture if the study was conducted in the West or the East, respectively. Prevalence of bullying was coded in frequencies. Sample sizes and statistic values $\left(\chi^{2}\right.$, means and standard deviations, $\mathrm{t}$ value and $p$ value, $\mathrm{F}$ value and $p$ value, correlation coefficients, standardized $\beta$ ) describing relationships between associated factors and bullying experiences were also coded. Consensus coding was made by two authors for each language. When information on sample characteristics, or statistic values were missing or unclear, the first author contacted and requested information from the lead author of the studies. Overall, 13 authors were contacted, and two provided requested information.

\section{STATISTICAL ANALYSIS}

Because the included studies varied significantly in their methods, analyses were conducted using a random effects model (Meta XL 5.3 and STATA 16.0), per the following procedure. Pooled prevalences, with 95\% confidence intervals (95\% CIs), of victimization, perpetration, and perpetration-victimization in general, verbal, physical, relational, and cyber forms were computed using the double arcsine transformation ${ }^{59}$ for students with ASD. When research participants responded 'yes' on any types of bullying questions or on a general question of being bullied by others or bullied others, it was categorized as "general" bullying. Their risk of bullying experiences was compared with that in typically developing students and students with other disabilities by calculating pooled odds ratios (ORs) with $95 \%$ CIs. Students with other disabilities were divided into nine groups, eight of which are based on the Individuals with Disabilities Education Act (IDEA) classifications ${ }^{60}$ as follows: hearing and visual impairment, emotional disturbance, intellectual disability, orthopedic impairment, other health impairment, specific learning disability, speech or language impairment, and traumatic brain injury. When type of disabilities was not available, they were assigned to the group of "various disabilities," the ninth group. Weighted mean effect sizes (pooled effect sizes) and 95\% CI were computed for degree of associations between associated factors and bullying experiences using Fisher's Zr transformations. Publication bias was assessed through visual inspection of Doi plots, as well as quantitative assessment of asymmetry through the LFK index. ${ }^{61}$ If the Doi plot indicated an existing asymmetry and the LFK index was $> \pm 1$, the presence of publication bias was suggested, indicating a possible skewing of the pooled results. Heterogeneity was investigated using Q statistics, and moderation analyses were conducted to identify sources of heterogeneity.

Efforts were made to avoid violation of the independence of observations assumption ${ }^{62}$ as follows. If multiple studies were published from the same data set $t^{20,23,39,53,54}$ and had multiple statistical values with the same information, multiple studies were considered as a single study and the highest value was selected. If statistical values of a parent variable (e.g., internalizing) and nested variables (e.g., fear, depression) for an associated factor were presented in a study, only the value of the parent variable was included. When there were multiple statistical values of nested variables (e.g., fear, depression) for an associated factor in a study, their average effect size was used.

Meta-analyses based on very small number of studies are subject to problems in statistical synthesis. ${ }^{63}$ Generally, it is recommended to include five or more studies for data synthesis, ${ }^{63}$ six or more studies for a continuous moderator, and four studies per level of a categorical moderator. ${ }^{64}$ However, due to the limited number of studies available for the current meta-analyses, we set the threshold of a minimum of three studies for pooled prevalence estimates, pooled ORs, and pooled effect sizes; a 
minimum of three studies for analyses using continuous moderators; and a minimum of three studies per level of analyses using categorical moderators. These criteria are consistent with those used in previous meta-analyses of a limited number of studies. ${ }^{65,66}$

\section{POOLED PREVALENCE ESTIMATES OF BULLYING IN STUDENTS WITH ASD}

Table 1 describes the results of pooled prevalence analyses. Supplementary Table 2 (only online) displays the forest plots and Doi plots of pooled prevalence estimates of bullying.

\section{Victimization}

The pooled prevalence for victimization in general was $67 \%$ (95\% CI $=57-76 \%)$. The prevalence of victimization for physical form was $30 \%$ (95\% CI=19-44\%), verbal form was 58\% (95\% $\mathrm{CI}=50-65 \%)$, relational form was $36 \%(95 \% \mathrm{CI}=22-51 \%)$, and cyber form was $15 \%(95 \% \mathrm{CI}=10-20 \%)$. There was considerable heterogeneity in every form of pooled victimization prevalence (general: $\mathrm{Q}=777.5, p<0.001$; physical: $\mathrm{Q}=141.6, p<0.001$; verbal: $\mathrm{Q}=85.4, p<0.001$, relational: $\mathrm{Q}=338.4, p<0.001$; cyber: $\mathrm{Q}=14.4$, $p=0.013)$. The Doi plot and LFK index suggested that publication bias was likely in all forms of victimization: general (major asymmetry, LFK=4.12), physical (major asymmetry, LFK=2.77), verbal (major asymmetry, LFK=-2.28), relational (major asymmetry, LFK=-2.62), and cyber (minor asymmetry, LFK=1.58) forms.

\section{Perpetration}

The pooled prevalence of perpetration in general was $29 \%$ (95\% CI=18-42\%). For physical, verbal, and relational forms of perpetration, prevalence estimates were $27 \%$ (95\% CI=12-46\%), $26 \%$ (95\% CI=4-56\%), and $12 \%$ (95\% CI=0-32\%), respectively. Pooled prevalence for cyber bullying was not computed because the number of studies that reported cyber-perpetration was less than three. All of the aforementioned estimates also showed considerable variability between studies (general: Q=
351.9, $p<0.001$; physical: $\mathrm{Q}=27.0, p<0.001$; verbal: $\mathrm{Q}=32.4, p<$ 0.001 ; relational: $\mathrm{Q}=19.5, p<0.001)$. Additionally, the Doi plot and LFK index indicated the presence of publication bias for general (major asymmetry, LFK=5.67), physical (major asymmetry, LFK=-2.79), and verbal forms (minor asymmetry, LFK= 1.29). No evidence of publication bias was found for the relational form (no asymmetry, LFK=0.46).

\section{Perpetration-victimization}

The pooled prevalence of perpetration-victimization in general was $14 \%(95 \% \mathrm{CI}=8-22 \%)$. There was a considerable amount of heterogeneity between studies $(\mathrm{Q}=45.3, p<0.001)$, as well as presence of publication bias based on major asymmetry of the Doi plot on visual inspection, coupled with the high LFK index (4.68). Pooled prevalence for each type was not computed due to a lack of data in the included studies.

\section{RISK OF VICTIMIZATION, PERPETRATION, AND PERPETRATION-VICTIMIZATION}

Pooled ORs for bullying in students with ASD were computed in comparison to typically developing students or students with other disabilities, including emotional disturbance, intellectual disability, other health problems, specific learning disability, and various disabilities (Table 2). Students with ASD faced a significantly higher risk for victimization than typically developing students $(\mathrm{OR}=2.35$; 95\% $\mathrm{CI}=1.57-3.53 ; p<0.001)$ and students with various disabilities $(\mathrm{OR}=1.98 ; 95 \% \mathrm{CI}=1.30-3.02$; $p=0.001$ ). On the other hand, their risk for victimization was not significantly different from students with emotional disturbance, intellectual disability, other health impairment, or specific learning disability. Pooled ORs for perpetration in students with ASD relative to both typically developing students and students with other health impairment were not significant.

Table 1. Pooled Prevalence of Bullying and Test Results for Heterogeneity and Publication Bias

\begin{tabular}{|c|c|c|c|c|c|c|}
\hline \multirow{2}{*}{ Bullying } & \multirow{2}{*}{$\begin{array}{c}\text { Bullying } \\
\text { forms }\end{array}$} & \multirow{2}{*}{$\begin{array}{l}\text { Number of } \\
\text { students }\end{array}$} & \multirow{2}{*}{$\begin{array}{l}\text { Number of } \\
\text { studies }\end{array}$} & \multirow{2}{*}{$\begin{array}{l}\text { Pooled prevalence }(\%) \\
(95 \% \mathrm{CI})\end{array}$} & Heterogeneity & \multirow{2}{*}{$\begin{array}{l}\text { Publication bias } \\
\text { Asymmetry (LFK) }\end{array}$} \\
\hline & & & & & Q statistics $(p)$ & \\
\hline \multirow[t]{5}{*}{ Victimization } & General & 2298 & 22 & $67(57-76)$ & $777.5(<0.001)$ & Major (4.12) \\
\hline & Physical & 287 & 10 & $30(19-44)$ & $141.6(<0.001)$ & Major (2.77) \\
\hline & Verbal & 978 & 12 & $58(50-65)$ & $85.4(<0.001)$ & Major (-2.28) \\
\hline & Relational & 822 & 12 & $36(22-51)$ & $338.4(<0.001)$ & Major (-2.62) \\
\hline & Cyber & 83 & 6 & $15(10-20)$ & $14.4(0.013)$ & Minor (1.58) \\
\hline \multirow[t]{4}{*}{ Perpetration } & General & 614 & 10 & $29(18-42)$ & $351.9(<0.001)$ & Major (5.67) \\
\hline & Physical & 83 & 4 & $27(12-46)$ & $27.0(<0.001)$ & Major (-2.79) \\
\hline & Verbal & 65 & 3 & $26(4-56)$ & $32.4(<0.001)$ & Minor (1.29) \\
\hline & Relational & 25 & 3 & $12(0-32)$ & $19.5(<0.001)$ & None (0.46) \\
\hline Perp-Vict & General & 175 & 4 & 14 (8-22) & $45.3(<0.001)$ & Major (4.68) \\
\hline
\end{tabular}

$\mathrm{Cl}$, confidence interval; Perp-Vict, Perpetration-Victimization. 


\section{FACTORS ASSOCIATED WITH BULLYING IN STUDENTS WITH ASD}

Pooled effect sizes for the associations between factors and bullying were computed when meeting statistical synthesis criteria ( $\geq 3$ studies). In result, eight factors for victimization (age, gender, ASD symptoms, deficits in social communication and social interaction, restricted, repetitive patterns of behavior, interests, or activities, externalizing symptoms, internalizing symptoms, inclusive school setting) and three factors for perpetration (gender, externalizing symptoms, internalizing symptoms) were included in this meta-analysis (Table 3). The forest plots and Doi plots are available in Supplementary Table 3 (only online).

Victimization was positively associated with deficits in social interaction and communication, externalizing symptoms, and internalizing symptoms. Interestingly, a positive effect size was found for victimization and the inclusive school setting: this means that students with ASD who were integrated in the inclusive school setting experienced more severe victimization than those who were not. Perpetration was positively associated with externalizing symptoms. A significant heterogeneity between studies was noted for all pooled effect sizes, with an exception in two pooled effect sizes (gender and perpetration, internalizing symptoms and perpetration). Also, Doi plots and the LFK index suggested that most of the pooled effect sizes had publication bias (minor to major asymmetry, LFK=1.174.83), except for three (gender and victimization, gender and perpetration, restricted, repetitive patterns of behavior, interests, or activities and victimization; no asymmetry, LFK=0.08-0.53).

\section{MODERATION ANALYSES}

Moderation analyses of pooled prevalence estimates

Due to a limited number of studies available for meta-analysis, moderation analyses of culture, school settings, and method of bullying measurement could not be performed to estimate pooled prevalences of perpetration in all forms. For the same reason, moderation analysis of culture was conducted only for victimization in general: this analysis included prevalence of verbal-victimization in an English study conducted in the East. ${ }^{40}$ Results are summarized in Tables 4 and 5, and the forest plots and Doi plots are illustrated in Supplementary Tables 4, 5, and 6 (only online).

Moderation analyses of the pooled prevalence of victimization revealed significant differences in culture (general: $\mathrm{R}^{2}=$

Table 2. Risk of Victimization, Perpetration, and Perpetration-Victimization

\begin{tabular}{llcccc}
\hline \multicolumn{1}{c}{ Disability types } & Bullying & Number of students & Number of studies & OR (95\% CI) & p value \\
\hline Typically developing & Victimization & 25123 & 7 & $2.35(1.57-3.53)$ & $1.10(0.33-3.63)$ \\
& Perpetration & 26392 & 4 & $0.51(0.25-1.06)$ & 0.881 \\
Emotional disturbance & Victimization & 1398 & 3 & $1.06(0.62-1.84)$ & 0.070 \\
Intellectual disability & Victimization & 1425 & 4 & $0.79(0.56-1.12)$ & $0.67(0.28-1.57)$ \\
Other health impairment & Victimization & 1968 & 3 & $0.96(0.77-1.20)$ & 0.179 \\
Specific learning disability & Perpetration & 5095 & 3 & $1.98(1.30-3.02)$ & 0.732 \\
Various disabilities & Victimization & 2595 & 3 & 0.001 \\
\hline
\end{tabular}

OR, odds ratio; $\mathrm{Cl}$, confidence interval.

Students with various disabilities: a group of students who have various types of disability.

Table 3. Pooled Effect Sizes and Test Results of Heterogeneity and Publication Bias

\begin{tabular}{|c|c|c|c|c|c|c|c|}
\hline \multirow{2}{*}{ Factor } & \multirow{2}{*}{ Bullying } & \multirow{2}{*}{$\begin{array}{l}\text { Number of } \\
\text { students }\end{array}$} & \multirow{2}{*}{$\begin{array}{l}\text { Number of } \\
\text { studies }\end{array}$} & \multirow{2}{*}{$\begin{array}{l}\text { Pooled } \\
(95 \% \text { CI) }\end{array}$} & \multirow{2}{*}{$p$ value } & Heterogeneity & \multirow{2}{*}{$\begin{array}{l}\text { Publication bias } \\
\text { Asymmetry (LFK) }\end{array}$} \\
\hline & & & & & & O statistics $(p)$ & \\
\hline Age & Victimization & 666 & 6 & $0.09(-0.11-0.28)$ & 0.386 & $33.2(<0.001)$ & Minor (1.89) \\
\hline \multirow[t]{2}{*}{ Gender } & Victimization & 3580 & 7 & $0.08(-0.01-0.16)$ & 0.081 & $32.3(<0.001)$ & None (0.08) \\
\hline & Perpetration & 2195 & 3 & $0.002(-0.04-0.04)$ & 0.912 & $1.7(0.422)$ & None (0.53) \\
\hline ASD symptoms & Victimization & 388 & 5 & $0.06(-0.17-0.28)$ & 0.605 & $18.5(0.001)$ & Minor (-1.56) \\
\hline SCl & Victimization & 708 & 7 & $0.35(0.22-0.47)$ & $<0.001$ & $10.0(0.004)$ & Major (2.54) \\
\hline RRB & Victimization & 188 & 3 & $0.11(-0.29-0.48)$ & 0.203 & $15.5(<0.001)$ & None $(0.16)$ \\
\hline \multirow[t]{2}{*}{ Externalizing symptoms } & Victimization & 2119 & 6 & $0.45(0.18-0.65)$ & 0.001 & $162.4(<0.001)$ & Major (4.48) \\
\hline & Perpetration & 1287 & 3 & $0.25(0.07-0.41)$ & 0.007 & $8.0(0.018)$ & Major (3.07) \\
\hline \multirow[t]{2}{*}{ Internalizing Symptoms } & Victimization & 1675 & 9 & $0.37(0.22-0.51)$ & $<0.001$ & $55.0(<0.001)$ & Major (4.83) \\
\hline & Perpetration & 1287 & 3 & $0.06(-0.03-0.14)$ & 0.203 & $2.6(0.275)$ & Minor (-1.17) \\
\hline Inclusive school setting & Victimization & 1408 & 6 & $0.34(0.05-0.58)$ & 0.025 & $117.8(<0.001)$ & Major (3.10) \\
\hline
\end{tabular}

$\mathrm{OR}$, odds ratio; $\mathrm{Cl}$, confidence interval; $\mathrm{ASD}$, autism spectrum disorder; $\mathrm{SCl}$, deficits in social interaction and communication; RRB, restricted, repetitive patterns of behavior, interests, or activities. 


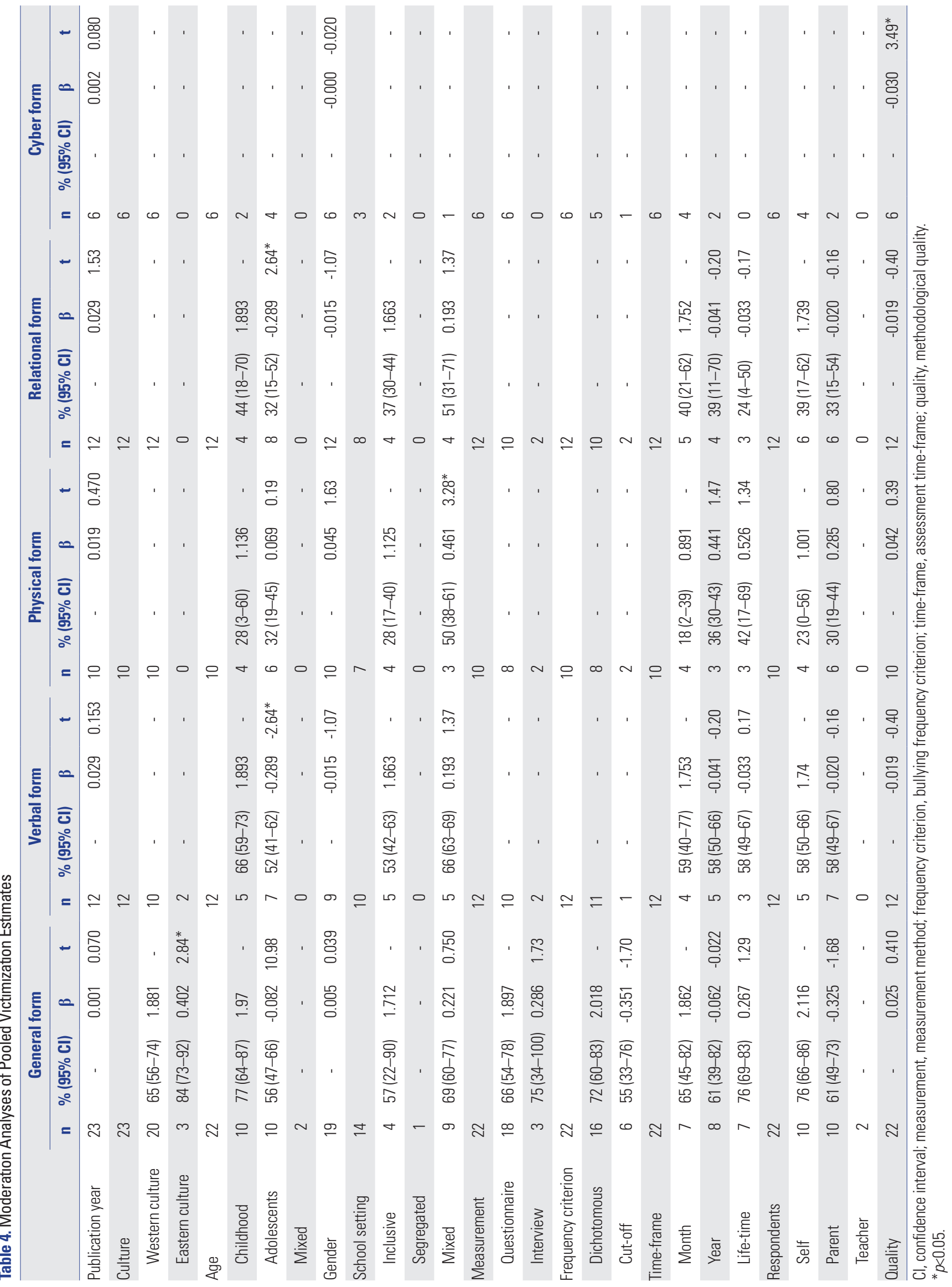


Table 5. Moderation Analysis of Pooled Perpetration Estimates

\begin{tabular}{|c|c|c|c|c|c|c|c|c|c|c|c|c|c|c|c|c|}
\hline \multirow{2}{*}{ Moderator } & \multicolumn{4}{|c|}{ General form } & \multicolumn{4}{|c|}{ Verbal form } & \multicolumn{4}{|c|}{ Physical form } & \multicolumn{4}{|c|}{ Relational form } \\
\hline & n & $\%(95 \%$ CI) & $\beta$ & $t$ & $n$ & $\%(95 \% \mathrm{CI})$ & $\beta$ & $t$ & $\mathbf{n}$ & $\%(95 \% \mathrm{Cl})$ & $\beta$ & $t$ & $\mathbf{n}$ & $\%(95 \% \mathrm{Cl})$ & $\beta$ & $t$ \\
\hline Publication year & 10 & - & -0.016 & -0.05 & 3 & - & 0.001 & 0.010 & 4 & - & 0.036 & 0.550 & 3 & - & -0.103 & -1.62 \\
\hline Age & 10 & & & & 3 & & & & 4 & & & & 3 & & & \\
\hline Childhood & 5 & $26(9-46)$ & 1.076 & - & 1 & - & - & - & 1 & - & - & - & 1 & - & - & - \\
\hline Adolescents & 4 & $30(12-51)$ & 0.091 & 0.32 & 2 & - & - & - & 3 & - & - & - & 2 & - & - & - \\
\hline Mixed & 1 & - & - & - & 0 & - & - & - & 0 & - & - & - & 0 & - & - & - \\
\hline Gender & 10 & - & 0.031 & 1.10 & 3 & - & -0.034 & -1.80 & 4 & - & -0.027 & -2.41 & 3 & - & 0.045 & 1.86 \\
\hline Frequency criterion & 10 & & & & 3 & & & & 4 & & & & 3 & & & \\
\hline Dichotomous & 6 & $26(14-40)$ & 1.079 & - & 3 & - & - & - & 4 & - & - & - & 3 & - & - & - \\
\hline Cut-off & 4 & $35(19-53)$ & 0.195 & 0.71 & 0 & - & - & - & 0 & - & - & - & 0 & - & - & - \\
\hline Time-frame & 10 & & & & 3 & & & & 4 & & & & 3 & & & \\
\hline Month & 5 & $26(7-50)$ & 1.091 & - & 2 & - & - & - & 3 & - & - & - & 2 & - & - & - \\
\hline Year & 4 & $31(12-53)$ & 0.096 & 0.32 & 1 & - & - & - & 1 & - & - & - & 1 & - & - & - \\
\hline Life-time & 1 & - & - & - & 0 & - & - & - & 0 & - & - & - & 0 & - & - & - \\
\hline Respondents & 10 & & & & 3 & & & & 4 & & & & 3 & & & \\
\hline Self & 3 & $34(16-53)$ & 1.242 & - & 1 & - & - & - & 2 & - & - & - & 1 & - & - & - \\
\hline Parent & 5 & $20(9-34)$ & -0.301 & -1.15 & 2 & - & - & - & 2 & - & - & - & 2 & - & - & - \\
\hline Teacher & 2 & - & - & - & 0 & - & - & - & 0 & - & - & - & 0 & - & - & - \\
\hline Quality & 10 & - & -0.129 & -1.45 & 3 & - & -0.379 & -2.06 & 4 & - & -0.050 & -0.21 & 3 & - & 0.810 & 0.57 \\
\hline
\end{tabular}

$\mathrm{Cl}$, confidence interval; frequency criterion, bullying frequency criterion; time-frame, assessment time-frame; quality, methodological quality.

0.093, $p=0.010$ ), age (verbal form: $\mathrm{R}^{2}=0.418, p=0.025$; relational form: $\mathrm{R}^{2}=0.418, p=0.025$ ), school settings (physical: $\mathrm{R}^{2}=0.684$, $p=0.022$ ), and methodological quality (cyber: $\mathrm{R}^{2}=0.077, p=0.025$ ). However, there were no significant differences in the pooled prevalences for victimization types by publication year, gender, bullying measurement method, bullying frequency criterion, assessment time-frame, or respondents (Table 4). No significant moderation effects were found in pooled prevalence for perpetration (Table 5).

On closer inspection, moderation analyses revealed that prevalence estimates for victimization in general were higher in Eastern culture than in Western culture. Prevalence estimates for verbal and relational-victimization were lower in adolescents than in children. Prevalence for physical-victimization was higher in the mixed school setting (inclusive setting+segregated setting) than the inclusive school setting. Finally, the higher methodological quality score was, the lower the prevalence of cyber-victimization was.

\section{Moderation analyses of pooled effect sizes}

The limited number of studies available for meta-analysis only allowed for moderation analyses of pooled effect sizes for victimization according to publication year, age, gender, respondents, and methodological quality. For the same reason, moderation analyses for perpetration were possible only in relation to publication year, gender, and methodological quality. The results are presented in Table 6.

Publication year moderated the relationship between internalizing symptoms and perpetration $\left(\mathrm{R}^{2}=0.995, p=0.028\right)$, and methodological quality was a significant moderator for the relationships between gender and perpetration $\left(\mathrm{R}^{2}=0.975, p=\right.$ $0.030)$, as well as internalizing symptoms and perpetration $\left(\mathrm{R}^{2}=\right.$ $0.992, p=0.049)$. Specifically, internalizing symptoms were less associated with perpetration in more recently published studies. Also, a higher methodological quality score was associated with a stronger association between gender (percentage of boys in the sample) and perpetration, as well as a weaker association between internalizing symptoms and perpetration.

\section{METHODOLOGICAL QUALITY OF THE REVIEWED STUDIES}

Maximum quality scores (14) were not achieved for any study included in the current meta-analysis. Most studies (97\% ; see Item 2. Study design) used an appropriate study design for their purposes. A few studies reported the methods used to determine the sample size (11\%; see Item 3. Sample Size Justification) and defined the population from which study subjects were selected (9\%; see Item 5. Sampling Frame). Many studies (80\%; see Item 8. Measurement Validity) used appropriate measurements. In most studies, the main findings were discussed in detail (71\%; see Item 17. Justified Discussion), and there were no concerns identified for funding sources or conflicts of interest affecting study results (94\%; see Item 19. Conflicts of Interest). Ethical consent from participants was reported to have been obtained in most studies (94\%; see Item 20. Ethical Approvals). In the assessment of bias, only a few studies (14\%; see Item 6, 


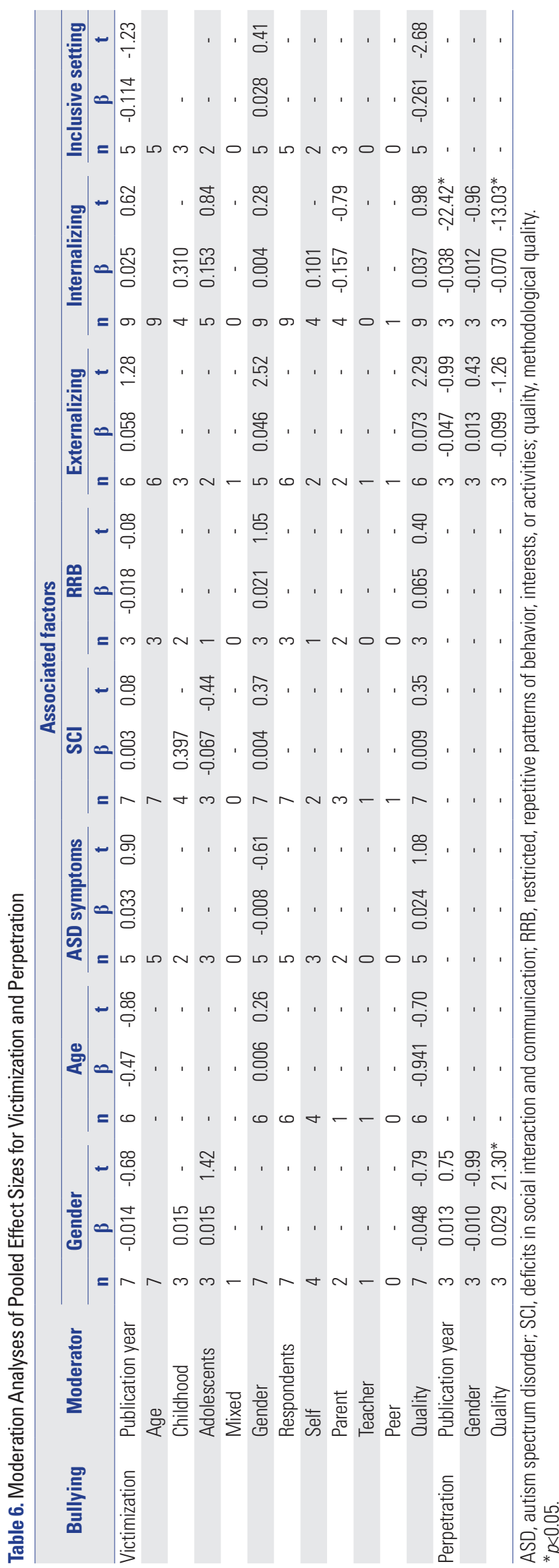

Sample Selection) used randomly selected population samples, leaving a potential for sampling bias in the rest of the studies in this meta-analysis. However, non-responders were addressed and categorized (e.g., quantifying non-responders, using a statistical method to address missing data); numbers of non-responders were modest, minimizing potentials to introduce a bias; and information about non-responders was described in many studies ( $74 \%$; see Item 7 . Non-responders, $77 \%$; see Item 13. Response Rate, $77 \%$; see Item 14 . Non-responders Information). In sum, the potential for non-responder bias was modest in the included studies. Half of the studies used measurements for which reliability had been verified previously (54\%; see Item 9. Measurement Reliability), leaving the other half vulnerable to information bias. Confounding factors were considered and controlled in more than half of the studies (63\%; see Item 11 . Confounding), but not in some studies, leaving the potential for confounding bias. Finally, most studies provided internally consistent results (89\%; see Item 15. Consistent Results), such that the risk of bias from inconsistent results was likely to be low (Supplementary Table 7, only online). ${ }^{33-56}$

\section{DISCUSSION}

Students with ASD face considerable difficulty in reciprocal social interactions, impairment in social communication skills, ${ }^{8}$ challenges in peer relationships, ${ }^{67,68}$ and emotional-behavioral problems ${ }^{69}$ These difficulties make them vulnerable to peer victimization. ${ }^{21,50}$ This study sought to outline the bullying experiences of students with ASD and factors associated therewith through a meta-analysis of published studies in order to suggest guidelines for clinical care for students with ASD and to provide directions for future studies.

Pooled prevalence estimates of victimization, perpetration, and perpetration-victimization in students with ASD were $67 \%$, $29 \%$, and $14 \%$, respectively, much higher than those for typically developing 6th-12th graders in the US (prevalences of $20 \%$, $19 \%$, and $7 \%$, respectively). ${ }^{70}$ They are also higher than those for victimization (36\%) and somewhat lower than those for perpetration (35\%) in typically developing adolescents reported in a meta-analysis of 80 studies published in English. ${ }^{71}$ Risk for victimization in students with ASD was 2.4 times higher than that for typical developing students and two times higher than that for students with various disabilities. The risk for perpetration was not different from that of typical developing students and students with other health impairment. These findings suggest that students with ASD are at higher risks for experiencing bullying, in particular victimization, when compared to typically developing children and those with other disabilities.

Regarding victimization, students with ASD experienced the verbal form most frequently (58\%), followed by relational (36\%), physical (30\%), and cyber-victimization (15\%), suggesting that a special focus on prevention of and intervention in verbal and 
relational bullying leaving no traces is necessary in this population. Students with ASD were less likely to be exposed to cyber-victimization than other traditional forms of victimization. However, $15 \%$ is never a small figure. The prevalence of cyberbullying for children with ASD will likely increase and become a greater focus in the future, as the number of students with ASD are increasingly using electronic devices and social networking services $^{24,72}$ due to their own interests and as a result of the COVID pandemic, which has forced increases in internet-based schooling and therapy services, as well as social isolation due to "sheltering-in-place." These circumstances should lead to the prompt initiation of preventive measures and interventions for cyber-victimization.

In regards to perpetration, physical-perpetration was most common (27\%), followed by verbal ( $26 \%$ ) and relational ( $12 \%)$ forms in students with ASD. Given their core deficit in social communication, it is not surprising that physical and verbal forms of perpetration are more common than relational forms in students with ASD. ${ }^{73,74}$ Some studies suggest that perpetration behaviors in students with ASD are related to comorbid conditions, ${ }^{16,40}$ suggesting clinical care for comorbid conditions and teaching coping skills to manage negative feelings and frustration are beneficial for preventing and/or reducing perpetration behaviors in students with ASD.

Social difficulties in students with ASD were positively related to bullying experience. Difficulties in forming positive peer relationships, as well as the lack of skills with which to cope with peer conflicts, might contribute to increased risks for bullying experiences. ${ }^{75,76}$ Comorbid conditions in students with ASD also are correlated with risks for being involved in bullying experiences. Specifically, externalizing symptoms are significantly correlated with victimization and perpetration, whereas internalizing symptoms are significantly correlated with victimization. The disruptive nature of these comorbid conditions may cause peer rejection and retaliation, as well as convey heightened vulnerabilities, in students with ASD, leading to an increased risk for victimization. ${ }^{77}$ Additionally, emotional outbursts or aggressive behavior, symptoms of externalizing behaviors, may be considered as perpetration behaviors. ${ }^{78}$

While comorbid conditions in children with ASD increase the risk for bullying experiences, it has also been established that bullying is related to later development of psychopathology. ${ }^{79,80}$ One study reported that $45 \%$ of adults with Asperger's syndrome had long-term sequelae from prior bullying experiences. ${ }^{81}$ Another study reported increased levels of anxiety in individuals with ASD who experience bullying victimization. ${ }^{22}$ A vicious cycle between bullying and psychosocial problems exists. ${ }^{82}$ Therefore, careful attention must be given to the children with ASD in order to protect these already vulnerable children from long-term consequences of bullying.

Inclusive school settings are significantly correlated with increases in victimization for students with ASD. Bullying occurs in the presence of a power imbalance between bullies and victims. ${ }^{83}$ Inherent power imbalance exists between students with ASD and typically developing peers due to impairments in social communication, social skills, and comorbid conditions in students with ASD. Our findings indicate that simply exposing students with ASD to inclusive school settings, without appropriate preparation, not only fails to promote acquisition of social skills and development of peer relationships for students with ASD, but also it poses harm to students with ASD by increasing their risks for peer victimization. For successful outcomes in inclusive education for students with ASD, as well as typically developing children, ongoing efforts are required for peer-mediated interventions against bullying, ${ }^{84}$ disability awareness education for students, families and school staff; ${ }^{85}$ supervision during recess time; and firm and consistent disciplinary methods for bullying across various settings, including homes, schools, and communities. ${ }^{86}$

Consistent with previous systematic review articles, ${ }^{14,31,32}$ we also identified significant heterogeneity within the included studies. Such heterogeneity may distort estimates of pooled prevalence and effect sizes in a meta-analysis. In order to mitigate the impact of heterogeneity, we used a random effects model in analyses to allow for harmonization of heterogenity of results between studies. ${ }^{64}$ We then conducted moderation analyses to identify sources of heterogeneity. The moderation analyses showed that the correlation between internalizing symptoms and perpetration is weaker in more recent studies. This may be due to differences in study methods over the time. The prevalence of general bullying was significantly higher in Eastern rather than Western culture. Eastern culture is characterized as collectivistic, with Western culture being more individualistic. In comparison to individualist culture, interdependency, cohesion, and unity are regarded as important values of the group in collective cultures. ${ }^{87}$ As such, the desire to follow collective norms to ensure harmony is the major force regulating behavior in Eastern cultures. ${ }^{88}$ Thus, it is plausible that peers in collective cultures may have rejected and bullied students with ASD who are different from them. In addition, inclusive education has been adopted in Eastern countries later than in Western countries, thus less experienced teachers, school personnel, and policymakers with respect to inclusive education might face more challenges in bullying prevention and intervention.

Victimization in verbal and relational bullying types was more frequent in younger students with ASD (5-12 years) than in adolescents with ASD (13-22 years). This pattern is consistent with that in typically developing students. For example, victimization was more frequent in 6th grade (approximately 12 years old) than in 7 th-10th grade students (approximately 13-16 years) in a survey of 6th-10th graders in schools from all areas of the US. ${ }^{89} \mathrm{~A}$ Korean study also reported that victimization in students with other disabilities was more frequent in elementary schools (approximately 8-12 years) than in high schools (approximately $16-18$ years). ${ }^{90}$ Fewer comorbid conditions (e.g., internalizing and externalizing symptoms) in older children 
with ASD than in younger children with ASD, ${ }^{91}$ along with more coping strategies developed with longer educational and social learning experiences, ${ }^{92}$ might have resulted in such an age pattern.

Students with ASD were significantly more likely to be targets of physical-victimization when schooled in the mixed school setting (inclusive setting+segregated setting) than in the inclusive school setting. Since sophisticated social communication skills are necessary to use relational aggression successfully, ${ }^{73,74}$ students with other disabilities included in segregated school settings may use physical aggression more frequently than relational aggression towards students with ASD due to their deficits in social communication skills.

Of the study methods, no moderators (bullying measurement method, criteria for prevalence estimates, assessment timeframe, respondents) had a significant effect on bullying prevalence. However, methodological quality had a significant impact on the prevalence of cyber-victimization and relationships between gender and perpetration and between internalizing and perpetration. Additionally, sampling problems (i.e., sample size justification, representativeness of the study subjects for target population) were common in most of the studies. Future studies need to make efforts to improve the methodological quality of their research and to minimize the possibility of bias stemming from sampling processes.

As in prior meta-analytic studies. ${ }^{14,65,93}$ Doi plots and LFK indices indicated publication bias in some estimates of pooled prevalence and effect sizes in our study. While this suggests that publication bias may have skewed the pooled estimates, it is difficult to adjust for publication bias in computing estimates of pooled prevalence and pooled effect sizes in the presence of between-study heterogeneity. ${ }^{94,95}$ Independent replications of our findings are warranted when more studies are accumulated and when methods for dealing with publication bias are advanced.

The limitations of this study include the following: 1) The small number of studies included in the current meta-analysis did not allow for the examination of the impact of more moderators and factors associated with bullying experiences. 2) The limited number of studies conducted in Eastern cultures makes it challenging to fully explore the impact of cultural differences on bullying in our study population. 3) Heterogeneity and publication bias were identified and may affect some estimates of pooled prevalence and effects sizes. Notwithstanding, our findings indicate that students with ASD are at higher risks for victimization and perpetration of bullying. The severity of victimization was higher when students with ASD were younger and were in inclusion classroom settings. Special attention is warranted to assist and/or promote adjustment of young students with ASD when they are placed in inclusion classroom settings. Clinicians, parents, teachers, and school personnel (including principles and school nurses) must be attentive when students with ASD present with high levels of social difficulties and in- ternalizing and/or externalizing symptoms. This should lead to assessments for whether the students with ASD are experiencing bullying. These symptoms are risk factors requiring increased attention in order to protect students with ASD from bullying and its detrimental consequences. Universal interventions for both students with ASD and other members of the school community, including classmates, teachers, and school personnel are crucial. Clear rules and expectations, as well as specific intervention protocols, about bullying should be established for all students, teachers, and school personnel. Bullying prevention education should be provided regularly for all. Further studies are warranted to identify other factors associated with bullying experiences in students with ASD and what prevention and/or intervention practices are effective in reducing this all-too-common form of school violence worldwide.

\section{AUTHOR CONTRIBUTIONS}

Conceptualization: Inhwan Park. Data curation: Inhwan Park, Jared Gong, Tomoya Hirota, Michio Takahashi, and Bora Kim. Formal analysis: Inhwan Park, Jared Gong, and Gregory L. Lyons. Investigation: Inhwan Park, Jared Gong, Tomoya Hirota, Michio Takahashi, and Bora Kim. Methodology: Inhwan Park and Gregory L. Lyons. Project administration: Inhwan Park. Resources: Inhwan Park. Software: Inhwan Park and Jared Gong. Supervision: Young Shin Kim. Validation: Seung-yeon Lee. Visualization: Inhwan Park and Jared Gong. Writing —original draft: Inhwan Park, Gregory L. Lyons, and Young Shin Kim. Writing-review \& editing: Inhwan Park, Young Shin Kim, Seungyeon Lee, Bennett L. Leventhal, and Jeongsoo Lee. Approval of final manuscript: all authors.

\section{ORCID iDs}

$\begin{array}{ll}\text { Inhwan Park } & \text { https://orcid.org/0000-0003-3575-9095 } \\ \text { Jared Gong } & \text { https://orcid.org/0000-0003-2876-2034 } \\ \text { Gregory L. Lyons } & \text { https://orcid.org/0000-0002-3697-2739 } \\ \text { Tomoya Hirota } & \text { https://orcid.org/0000-0002-2101-2655 } \\ \text { Michio Takahashi } & \text { https://orcid.org/0000-0002-7151-1364 } \\ \text { Bora Kim } & \text { https://orcid.org/0000-0002-6333-9023 } \\ \text { Seung-yeon Lee } & \text { https://orcid.org/0000-0002-2505-895X } \\ \text { Young Shin Kim } & \text { https://orcid.org/0000-0002-6766-1722 } \\ \text { Jeongsoo Lee } & \text { https://orcid.org/0000-0002-6731-2557 } \\ \text { Bennett L. Leventhal } & \text { https://orcid.org/0000-0001-6985-3691 }\end{array}$

\section{REFERENCES}

1. Parker JG, Rubin KH, Erath SA, Wojslawowicz JC, Buskirk AA. Peer relationships, child development, and adjustment: a developmental psychopathology perspective. In: Cicchetti D, Cohen DJ, editors. Developmental psychopathology: theory and method. Vol. 1. 2nd ed. Hoboken (NJ): John Wiley \& Sons. Inc.; 2006. p.419-93.

2. Golshiri P, Farajzadegan Z, Tavakoli A, Heidari K. Youth violence and related risk factors: a cross-sectional study in 2800 adolescents. Adv Biomed Res 2018;7:138.

3. Rose CA, Monda-Amaya LE, Espelage DL. Bullying perpetration and victimization in special education: a review of the literature. Remedial Spec Educ 2011;32:114-30. 
4. Bear GG, Mantz LS, Glutting JJ, Yang C, Boyer DE. Differences in bullying victimization between students with and without disabilities. School Psych Rev 2015;44:98-116.

5. Blake JJ, Lund EM, Zhou Q, Kwok OM, Benz MR. National prevalence rates of bully victimization among students with disabilities in the United States. Sch Psychol Q 2012;27:210-22.

6. Farmer TW, Lane KL, Lee DL, Hamm JV, Lambert K. The social functions of antisocial behavior: considerations for school violence prevention strategies for students with disabilities. Behav Disord 2012;37:149-62.

7. Mahady Wilton MM, Craig WM, Pepler DJ. Emotional regulation and display in classroom victims of bullying: characteristic expressions of affect, coping styles and relevant contextual factors. Soc Dev 2000;9:226-45.

8. Frith U, Hill EL. Autism: mind and brain. New York (NY): Oxford University Press; 2004.

9. Fox CL, Boulton MJ. The social skills problems of victims of bullying: self, peer and teacher perceptions. Br J Educ Psychol 2005;75: 313-28.

10. Gini G, Albiero P, Benelli B, Altoè G. Does empathy predict adolescents' bullying and defending behavior? Aggress Behav 2007; 33:467-76.

11. Bodfish JW. Repetitive behaviors in individuals with autism spectrum disorders. In: Amaral DG, Dawson G, Geschwind DH, editors. Autism spectrum disorders. New York (NY): Oxford University Press; 2011. p.200-12.

12. Reese RM, Richman DM, Belmont JM, Morse P. Functional characteristics of disruptive behavior in developmentally disabled children with and without autism. J Autism Dev Disord 2005;35:419-28.

13. Cook CR, Williams KR, Guerra NG, Kim TE, Sadek S. Predictors of bullying and victimization in childhood and adolescence: a metaanalytic investigation. Sch Psychol Q 2010;25:65-83.

14. Maïano C, Normand CL, Salvas MC, Moullec G, Aimé A. Prevalence of school bullying among youth with autism spectrum disorders: a systematic review and meta-analysis. Autism Res 2016; 9:601-15.

15. Kloosterman PH, Kelley EA, Craig WM, Parker JDA, Javier C. Types and experiences of bullying in adolescents with an autism spectrum disorder. Res Autism Spectr Disord 2013;7:824-32.

16. Montes G, Halterman JS. Bullying among children with autism and the influence of comorbidity with ADHD: a population-based study. Ambul Pediatr 2007;7:253-7.

17. Cappadocia MC, Weiss JA, Pepler D. Bullying experiences among children and youth with autism spectrum disorders. J Autism Dev Disord 2012;42:266-77.

18. Chiu YL, Kao S, Tou SW, Lin FG. Effects of heterogeneous risk factors on psychological distress in adolescents with autism and victimization experiences in Taiwan. Disabil Rehabil 2018;40:42-51.

19. Nowell KP, Brewton CM, Goin-Kochel RP. A multi-rater study on being teased among children/adolescents with autism spectrum disorder (ASD) and their typically developing siblings: associations with ASD symptoms. Focus Autism Other Dev Disabl 2014; 29:195-205.

20. Zablotsky B, Bradshaw CP, Anderson CM, Law P. Risk factors for bullying among children with autism spectrum disorders. Autism 2014;18:419-27.

21. Fink E, Olthof T, Goossens F, van der Meijden S, Begeer S. Bullying-related behaviour in adolescents with autism: links with autism severity and emotional and behavioural problems. Autism 2018;22:684-92.

22. Weiss JA, Cappadocia MC, Tint A, Pepler D. Bullying victimization, parenting stress, and anxiety among adolescents and young adults with autism spectrum disorder. Autism Res 2015;8:727-37.
23. Hebron J, Humphrey N. Exposure to bullying among students with autism spectrum conditions: a multi-informant analysis of risk and protective factors. Autism 2014;18:618-30.

24. Kuo MH, Orsmond GI, Coster WJ, Cohn ES. Media use among adolescents with autism spectrum disorder. Autism 2014;18:91423.

25. Han G, Park B. Children's choice in conflict: application of the theory of individualism-collectivism. J Cross Cult Psychol 1995; 26:298-313.

26. Kanetsuna T, Smith PK, Morita Y. Coping with bullying at school: children's recommended strategies and attitudes to school-based interventions in England and Japan. Aggress Behav 2006;32:57080.

27. Stone DL, Rosopa PJ. The advantages and limitations of using meta-analysis in human resource management research. Hum Resour Manag Rev 2017;27:1-7.

28. Humphrey N, Hebron J. Bullying of children and adolescents with autism spectrum conditions: a 'state of the field' review. Int J Incl Educ 2015;19:845-62.

29. Kim JW, Park JY. A study on research trends and victimization variables regarding students with disabilities who are vulnerable to school violence. J Dev Disabil 2014;18:125-55.

30. Lee HJ. An integrated literature review on bullying issues related to students with autism spectrum disorders. J Korean Assoc Persons Autism 2013;13:87-109.

31. Schroeder JH, Cappadocia MC, Bebko JM, Pepler DJ, Weiss JA. Shedding light on a pervasive problem: a review of research on bullying experiences among children with autism spectrum disorders. J Autism Dev Disord 2014;44:1520-34.

32. Sreckovic MA, Brunsting NC, Able H. Victimization of students with autism spectrum disorder: a review of prevalence and risk factors. Res Autism Spectr Disord 2014;8:1155-72.

33. Adams RE, Fredstrom BK, Duncan AW, Holleb LJ, Bishop SL. Using self- and parent-reports to test the association between peer victimization and internalizing symptoms in verbally fluent adolescents with ASD. J Autism Dev Disord 2014;44:861-72.

34. Adams R, Taylor J, Duncan A, Bishop S. Peer victimization and educational outcomes in mainstreamed adolescents with autism spectrum disorder (ASD). J Autism Dev Disord 2016;46:3557-66.

35. Bitsika V, Sharpley CF. Understanding, experiences, and reactions to bullying experiences in boys with an autism spectrum disorder. J Dev Phys Disabil 2014;26:747-61.

36. Campbell M, Hwang YS, Whiteford C, Dillon-Wallace J, Ashburner J, Saggers B, et al. Bullying prevalence in students with autism spectrum disorder. Australas J Spec Edu 2017;41:101-22.

37. Carter S. Bullying of students with Asperger syndrome. Issues Compr Pediatr Nurs 2009;32:145-54.

38. Chen PY, Schwartz IS. Bullying and victimization experiences of students with autism spectrum disorders in elementary schools. Focus Autism Other Dev Disabl 2012;27:200-12.

39. Hebron J, Oldfield J, Humphrey N. Cumulative risk effects in the bullying of children and young people with autism spectrum conditions. Autism 2017;21:291-300.

40. Hwang S, Kim YS, Koh YJ, Leventhal BL. Autism spectrum disorder and school bullying: who is the victim? Who is the perpetrator? J Autism Dev Disord 2018;48:225-38.

41. Rieffe C, Camodeca M, Pouw LBC, Lange AMC, Stockmann L. Don't anger me! Bullying, victimization, and emotion dysregulation in young adolescents with ASD. Eur J Dev Psychol 2012;9: 351-370.

42. Rose CA, Simpson CG, Moss A. The bullying dynamic: prevalence of involvement among a large-scale sample of middle and high school youth with and without disabilities. Psychol Sch 2015;52: 
515-31.

43. Rose CA, Stormont M, Wang Z, Simpson CG, Preast JL, Green AL. Bullying and students with disabilities: examination of disability status and educational placement. School Psych Rev 2015;44:42544.

44. Rowley E, Chandler S, Baird G, Simonoff E, Pickles A, Loucas T, et al. The experience of friendship, victimization and bullying in children with an autism spectrum disorder: associations with child characteristics and school placement. Res Autism Spectr Disord 2012;6:1126-34.

45. Sofronoff K, Dark E, Stone V. Social vulnerability and bullying in children with Asperger syndrome. Autism 2011;15:355-72.

46. Sterzing PR, Shattuck PT, Narendorf SC, Wagner M, Cooper BP. Bullying involvement and autism spectrum disorders: prevalence and correlates of bullying involvement among adolescents with an autism spectrum disorder. Arch Pediatr Adolesc Med 2012;166: 1058-64.

47. Storch EA, Larson MJ, Ehrenreich-May J, Arnold EB, Jones AM, Renno P, et al. Peer victimization in youth with autism spectrum disorders and co-occurring anxiety: relations with psychopathology and loneliness. J Dev Phys Disabil 2012;24:575-90.

48. Twyman KA, Saylor CF, Saia D, Macias MM, Taylor LA, Spratt E. Bullying and ostracism experiences in children with special health care needs. J Dev Behav Pediatr 2010;31:1-8.

49. Ung D, McBride N, Collier A, Selles R, Small B, Phares V, et al. The relationship between peer victimization and the psychological characteristics of youth with autism spectrum disorder. Res Autism Spectr Disord 2016;32:70-9.

50. van Roekel E, Scholte RH, Didden R. Bullying among adolescents with autism spectrum disorders: prevalence and perception. J Autism Dev Disord 2010;40:63-73.

51. van Schalkwyk G, Smith IC, Silverman WK, Volkmar FR. Brief report: bullying and anxiety in high-functioning adolescents with ASD. J Autism Dev Disord 2018;48:1819-24.

52. Wainscot JJ, Naylor P, Sutcliffe P, Tantam D, Williams JV. Relationships with peers and use of the school environment of mainstream secondary school pupils with Asperger syndrome (high-functioning autism): a case-control study. Rev Int Psicol Ter Psicol 2008;8: 25-38.

53. Zablotsky B, Bradshaw CP, Anderson C, Law PA. Involvement in bullying among children with autism spectrum disorders: parents' perspectives on the influence of school factors. Behav Disord 2012; 37:179-91.

54. Zablotsky B, Bradshaw CP, Anderson C, Law PA. The association between bullying and the psychological functioning of children with autism spectrum disorders. J Dev Behav Pediatr 2013;34:1-8.

55. Zeedyk SM, Rodriguez G, Tipton LA, Baker BL, Blacher J. Bullying of youth with autism spectrum disorder, intellectual disability, or typical development: victim and parent perspectives. Res Autism Spectr Disord 2014;8:1173-83.

56. Tada S, Sugiyama T, Nishizawa M, Tsujii S. A clinical research of teasing to high functioning pervasive developmental disorders. Psychiatr Neurol Paediatr Jpn 1998;38:195-204.

57. Downes MJ, Brennan ML, Williams HC, Dean RS. Development of a critical appraisal tool to assess the quality of cross-sectional studies (AXIS). BMJ Open 2016;6:e011458.

58. Center for Evidence Based Management. Critical appraisal checklist for cross-sectional study [accessed on 2017 November 1]. Available at: https://www.cebma.org/resources-and-tools/whatis-critical-appraisal/.

59. Barendregt JJ, Doi SA, Lee YY, Norman RE, Vos T. Meta-analysis of prevalence. J Epidemiol Community Health 2013;67:974-8.

60. The Individuals with Disabilities Education Act, 82 C.F.R. Sect.
300.8 (2017).

61. Furuya-Kanamori L, Barendregt JJ, Doi SAR. A new improved graphical and quantitative method for detecting bias in metaanalysis. Int J Evid Based Healthc 2018;16:195-203.

62. Tracz SM, Elmore PB, Pohlmann JT. Correlational meta-analysis: independent and non independent cases. Educ Psychol Meas 1992;52:879-88.

63. Davey J, Turner RM, Clarke MJ, Higgins JP. Characteristics of meta-analyses and their component studies in the Cochrane Database of Systematic Reviews: a cross-sectional, descriptive analysis. BMC Med Res Methodol 2011;11:160.

64. Kim SY, Park JE, Seo HJ, Lee YJ, Jang BH, Son HJ, et al. NECA's guidance for undertaking systematic reviews and meta-analyses for intervention. Seoul: National Evidence-Based Healthcare Collaborating Agency; 2011.

65. Compas BE, Jaser SS, Bettis AH, Watson KH, Gruhn MA, Dunbar JP, et al. Coping, emotion regulation, and psychopathology in childhood and adolescence: a meta-analysis and narrative review. Psychol Bull 2017;143:939-91.

66. Slagt M, Dubas JS, Deković M, van Aken MA. Differences in sensitivity to parenting depending on child temperament: a meta-analysis. Psychol Bull 2016;142:1068-110.

67. Jordan R. Social play and autistic spectrum disorders: a perspective on theory, implications and educational approaches. Autism 2003;7:347-60.

68. Koegel LK, Koegel RL, Frea WD, Fredeen RM. Identifying early intervention targets for children with autism in inclusive school settings. Behav Modif 2001;25:745-61.

69. Bauminger N, Kasari C. Loneliness and friendship in high-functioning children with autism. Child Dev 2000;71:447-56.

70. Carlyle KE, Steinman KJ. Demographic differences in the prevalence, co-occurrence, and correlates of adolescent bullying at school. J Sch Health 2007;77:623-9.

71. Modecki KL, Minchin J, Harbaugh AG, Guerra NG, Runions KC. Bullying prevalence across contexts: a meta-analysis measuring cyber and traditional bullying. J Adolesc Health 2014;55:602-11.

72. Mazurek MO. Social media use among adults with autism spectrum disorders. Comput Human Behav 2013;29:1709-14.

73. Björkqvist K, Österman K, Kaukiainen A. The development of direct and indirect aggressive strategies in males and females. In: Björkqvist K, Niemelä P, editors. Of mice and women: aspects of female aggression. San Diego (CA): Academic Press; 1992. p.51-64.

74. Kaukiainen A, Björkqvist K, Lagerspetz K, Österman K, Salmivalli C, Rothberg S, et al. The relationships between social intelligence, empathy, and three types of aggression. Aggress Behav 1999;25: 81-9.

75. Kochenderfer-Ladd B, Skinner K. Children's coping strategies: moderators of the effects of peer victimization? Dev Psychol 2002; 38:267-78.

76. Mouttapa M, Valente T, Gallaher P, Rohrbach LA, Unger JB. Social network predictors of bullying and victimization. Adolescence 2004;39:315-35.

77. Farmer TW, Petrin R, Brooks DS, Hamm JV, Lambert K, Gravelle M. Bullying involvement and the school adjustment of rural students with and without disabilities. J Emot Behav Disord 2012;20: 19-37.

78. Han SY. Communication with parents, self-esteem and shame and their relationship to aggression in bully and bully-victim groups. Korean J Hum Dev 2010;17:125-43.

79. Copeland WE, Wolke D, Angold A, Costello EJ. Adult psychiatric outcomes of bullying and being bullied by peers in childhood and adolescence. JAMA Psychiatry 2013;70:419-26.

80. Kim YS, Leventhal BL, Koh YJ, Boyce WT. Bullying increased sui- 
cide risk: prospective study of Korean adolescents. Arch Suicide Res 2009;13:15-30.

81. Samson AC, Huber O, Ruch W. Teasing, ridiculing and the relation to the fear of being laughed at in individuals with Asperger's syndrome. J Autism Dev Disord 2011;41:475-83.

82. Busch V, Laninga-Wijnen L, van Yperen TA, Schrijvers AJP, De Leeuw JR. Bidirectional longitudinal associations of perpetration and victimization of peer bullying with psychosocial problems in adolescents: a cross-lagged panel study. Sch Psychol Int 2015;36: 532-49.

83. Olweus D. Bullying at school: basic facts and effects of a school based intervention program. J Child Psychol Psychiatry 1994;35: 1171-90.

84. Watkins L, O'Reilly M, Kuhn M, Gevarter C, Lancioni GE, Sigafoos $\mathrm{J}$, et al. A review of peer-mediated social interaction interventions for students with autism in inclusive settings. J Autism Dev Disord 2015;45:1070-83.

85. Lindsay S, Edwards A. A systematic review of disability awareness interventions for children and youth. Disabil Rehabil 2013;35: 623-46.

86. Ttofi MM, Farrington DP. Effectiveness of school-based programs to reduce bullying: a systematic and meta-analytic review. J Exp Criminol 2011;7:27-56.

87. Yamaguchi S. Culture and control orientations. In: Matsumoto D, editor. The handbook of culture and psychology. New York (NY): Oxford University Press; 2001. p.223-43.

88. Triandis HC, McCusker C, Hui CH. Multimethod probes of individualism and collectivism. J Pers Soc Psychol 1990;59:1006-20.

89. Wang J, Iannotti RJ, Nansel TR. School bullying among adolescents in the United States: physical, verbal, relational, and cyber. J Adolesc Health 2009;45:368-75.

90. Song YB, Kwon SS, Lee YS. A study on bullying and students with disabilities in inclusive classrooms. J Incl Educ 2013;8:1-21.

91. Gray K, Keating C, Taffe J, Brereton A, Einfeld S, Tonge B. Trajectory of behavior and emotional problems in autism. Am J Intellect Dev Disabil 2012;117:121-33.

92. Lee KW, Jung SA. School violence experience and coping of students with high functioning autism spectrum disorders in inclusive education environment. J Korean Soc Integr Med 2016;4:6979 .

93. Dzhambov AM, Lercher P. Road traffic noise exposure and birth outcomes: an updated systematic review and meta-analysis. Int J Environ Res Public Health 2019;16:2522.

94. Shi L, Lin L. The trim-and-fill method for publication bias: practical guidelines and recommendations based on a large database of meta-analyses. Medicine (Baltimore) 2019;98:e15987.

95. Terrin N, Schmid CH, Lau J, Olkin I. Adjusting for publication bias in the presence of heterogeneity. Stat Med 2003;22:2113-26. 